The International Journal Of Engineering And Science (IJES)

|| Volume || 6 || Issue || 3 || Pages || PP 25-39 || 2017 ||

ISSN (e): $2319-1813 \operatorname{ISSN}$ (p): $2319-1805$

THE IJES

\title{
Application of Synchronized Phasor Measurements Units in Power Systems
}

\author{
Nikolaos. P. Theodorakatos \\ School of Electrical and Computer Engineering, National Technical University of Athens \\ Athens, 15780, Greece
}

\begin{abstract}
-ABSTRACT-
The last decades, electric power industry is undergoing multiple changes due to the process of deregulation, providing efficient power generation, technological innovations, and eventually lower retail prices. In this environment, dynamic phenomena in power systems have made ever more urgent the development of reliable tools for their monitoring and control. An effective tool for the close monitoring of their operation conditions is the state estimator. The traditional estimators are based on real time measurements obtained through SCADA (Supervisory Control and Data Acquisition) system. These measurements are commonly provided by the remote terminal units (RTUs) installed at the high voltage substations. The phase angle of bus voltages can not be easily measured due to technical difficulties associated with the synchronization of measurements at RTUs. Global Positioning System (GPS) alleviated these difficulties and led to the development of Phasor Measurement Units (PMUS). This weakness was eliminated with the arrival of GPS, which led to the development of Phasor Measurement Units. A PMU unit, equipped with a GPS receiver, provides high accuracy voltage and current phasor measurements with respect to a common reference phase angle. In the first part of the paper, an overview of the PMU technology and a review about the optimal allocation of PMUs in power network are presented. The most important issues regarding design and operation of PMUs are discussed and an analysis of their commercial penetration in the electric energy markets is made. The second part of the paper presents a wide range of applications related with the choice of the strategic PMU placement as well as an algorithm for finding the optimal number of PMUs needed for full observability.
\end{abstract}

Index Terms: Bibliographic Analysis, Optimal PMU Placement, Phasor Measurement Unit, synchronized measurements, Global Positioning System, Smart Grid, software applications, monitoring, system, architecture

Date of Submission: 16 February $2017 \quad$ Date of Accepted: 05 March 2017

\section{INTRODUCTION}

State estimation (SE) is a critical power system management application. The function of SE is needed to obtain the best estimate of the current state of an electric system, based on available redundant measurements, such as voltage magnitudes and real and reactive power flows and injections [1]. For an power system, these measurements are collected from devices well distributed over the system, digitized and, finally, transmitted to the central computer, whereas, for power system, the digital data are transmitted using fiber optics due to the high data rate, noise immunity, light weight, and electrical isolation. The state estimator accuracy, in power systems, is significantly improved by the inclusion of the more accurate and time time-tagged measurements provided by the phasor measurement units (PMUs). A PMU, when placed at a bus, can measure the voltage phasor of the installed bus and the current phasors of some or all the branches incident to the bus, assuming that the PMU has sufficient number of channels [2]-[5]. Nowadays, utilization of PMUs in the monitoring, protection and control of power systems has become increasingly important. The objective of the optimal PMU placement (OPP) problem is the determination of the minimum number and locations of PMUs ensuring the power system observability. Several methods have been proposed for the OPP problem. These methods are classified into two major categories: deterministic and heuristic algorithms. The first category includes optimization-based deterministic methods. The basic idea is to use the deterministic algorithm but starts with different initial points. Deterministic algorithms follow a rigorous procedure and its path and values of both design variables and the functions are repeatable. The basic idea is to use the deterministic algorithm, but start with different initial points. Deterministic algorithms including discrete and continuous programming techniques follow a rigorous procedure. On the other hand the stochastic algorithms always have some randomness solving a design which is an iterative process [6]. Both algorithms have been proposed and implemented for the solution of the optimal allocation of PMUs in a power network [7]. An integer programming formulation of OPP problem considering or not the existence of conventional measurements and ensuring network observability is proposed in [8], [9]. In [10], a comparative study is implemented between a 
deterministic technique and a heuristic method. The binary integer programming algorithm and a genetic algorithm has been used in order to solve the OPP problem as proposed in [9]. A multistage PMU placement procedure based on integer linear programming (ILP) and modeling the zero injections as linear constraints is suggested in [11]. Two indices, the bus observability index (BOI) and the system observability redundancy index (SORI), are also introduced to further rank the multiple solutions. For OPP problematic, the nonlinear programming and a sequential quadratic programming method are formulated using binary integer programming (BIP) model in [12]. Based on NLP the feasibility is investigated for obtaining optimal solution. The System Observability Redundancy Index as proposed in [11] is measured for ranking the multiple solutions. An integer linear programming placement algorithm, considering the full network observability with or without loss of a single transmission line or PMU as well as conventional measurements existence, is suggested in [13]. Sequential quadratic programming algorithm is adopted to solve the optimal allocation of PMUs in power networks in [14]-[15]. A wide range of metaheuristic optimization techniques have also been proposed [6]. A binary particle swarm optimization (BPSO) technique considering contingency conditions, i.e., PMU loss or branch outage, and ensuring network observability is suggested in [16]. Also, others non-deterministic algorithm such as Binary Search Algorithm (BSA) [17], Binary Particle Swarm Optimization (BPSO) [18], Tabu Search Algorithm (TSA) [19], and Recursive Tabu Search [19], have been used in solving the OPP problem. This paper is organized as follows : Section II discusses the fundamentals of PMUs. Section III discusses the PMU technology. In section IV, it is presented the synchrophasor standard IEEE C37.118. Section V presents the manufacturing companies of PMUs and the installation of these devices in power network for better and reliable monitoring in different countries around the world. The PMU placement optimization is discussed in section VI, whereas section VII presents a case study of the optimization of optimal allocation of PMUs applied to real power systems. In this section, a branch and bound algorithm is presented for the solution of the discrete optimization problem. In section VIII, it is presented the application of PMUs in modern power systems. In the existing power measurements, the available measurements are received by SCADA system, so the contribution of PMUs in the state estimator is presented. Section X presents existing synchrophasor installations in power networks in countries around the world. In section XI a phasor measurement application study is presented. Finally, Section XII concludes this paper.

\section{FUNDAMENDALS OF PMUS}

PMUs technology provides phasor information (both magnitude and phase angle) in real time [5]. The advantage of referring phase angle to a global reference time is helpful in capturing the wide area snap shot of the power system. Effective utilization of this technology is very useful in mitigating blackouts and learning the real time behaviour of the power system. With the advancement in technology, the micro processor based instrumentation such as protection Relays and Disturbance Fault Recorders (DFRs) incorporate the PMU module along with other existing functionalities as an extended feature [5]. A pure sinusoidal waveform can be represented by a unique complex number known as a phasor. Consider a sinusoidal signal [5] :

$$
y(t)=\hat{y} \cos (\omega t+\varphi)
$$

The phasor representation of this sinusoidal is given by [5] :

$$
\begin{aligned}
& y(t)=\operatorname{Re}\left[\hat{y} e^{j(\omega t+\varphi)}\right]=\operatorname{Re}\left[e^{j \omega t} \hat{y} e^{j \varphi}\right] \\
& y(t) \leftrightarrow Y=\frac{\hat{y}}{\sqrt{2}} e^{j \varphi}=\frac{\hat{y}}{\sqrt{2}}[\cos \varphi+j \sin \varphi]=\frac{\hat{y}}{\sqrt{2}} \angle \varphi
\end{aligned}
$$

The magnitude of the phasor is the rms value of the sinusoid $\frac{\hat{y}}{\sqrt{2}}$ and its phase is $\varphi$. The sinusoidal signal and its phasor representation given by (1) and (3) are illustrated in Fig. 1
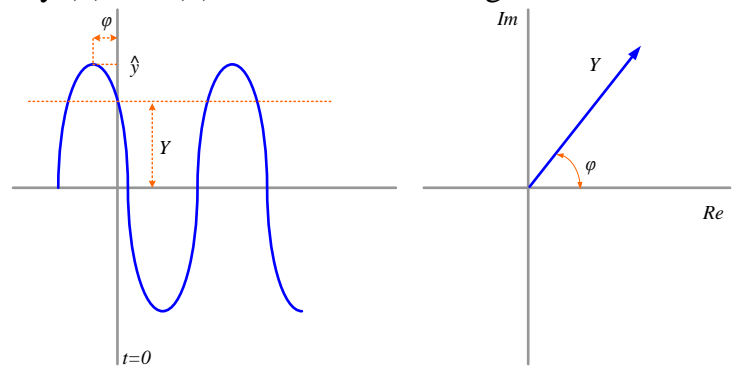

Fig. 1. Phasor representation of a sinusoidal signal. (a) Sinusoidal signal. (b) Phasor representation [5] 


\section{PMU TECHNOLOGY}

The first phasor measurement units (PMUs) built at the Power Systems Research Laboratory at Virginia Tech. The GPS receiver clock was external to the PMU, and with the small number of GPS satellites deployed at that time, the clock had to be equipped with a precision internal oscillator which maintained accurate time in the absence of visible satellites [5].

\section{III.1 PMU CONFIGURATION}

A PMU block diagram is shown in Figure 2.
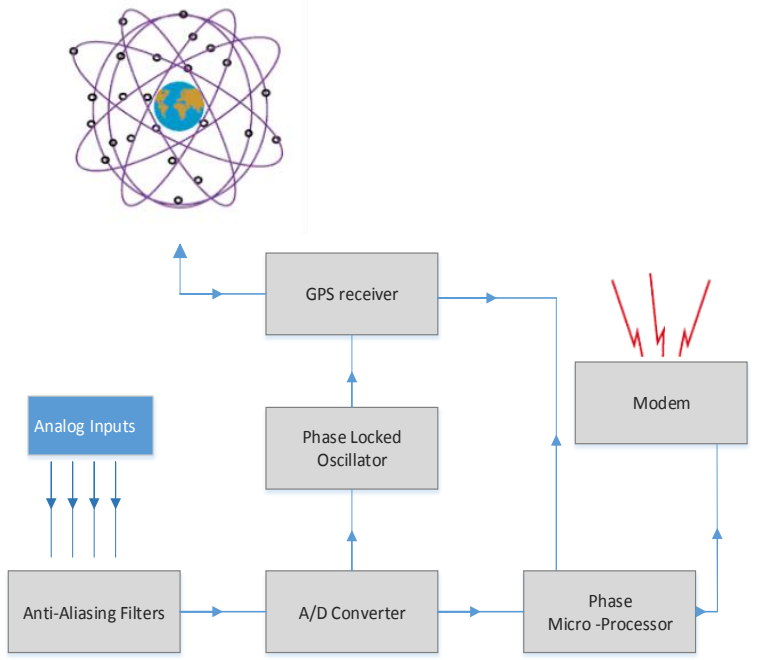

Figure 2. PMU configuration [5], [21]

Each block is detailed below [5]. A voltage or current level matched with the requirements of the converters are obtained by using appropriate measurement transformers. The analog $\mathrm{AC}$ waveforms are digitized by an Analog-to-Digital converter. The role of the anti-aliasing filter placed before the signal sampler is to remove the components of the signal whose frequency is equal or greater than one-half the Nyquiste (sample) rate. The sampling is done using the Phasor Measurement Algorithm based on the Discrete Fourier Transform (DFT). Let us assume that the sampling frequency is $F_{s}=N f$ where $f$ is the signal frequency, so this means that there are $N$ samples per cycle of the signal and the sampling of the signal over the window is denoted by the set. The variable $N$ is also called the window-length, equal to the number of samples per cycle. The sampling period is therefore $T=\frac{1}{f}$. The process of computing this phasor estimate requires sampling the waveform over some interval of time, which is the measurement window. The time at which the phasor is estimated is the phasor time-tag. DFT-based phasor calculation at a particular instant of time is $X=\frac{\sqrt{2}}{N} \sum_{k=0}^{N-1} x_{k} e^{-j \omega T k}$. Estimating the phasor requires sampling the waveform over some interval of time [23]. An important point discussed in the standard is the choice of the time-tag within the window as shown in Figure 3 whereas Fig 4 shows a PMU devices with GPS receiver.

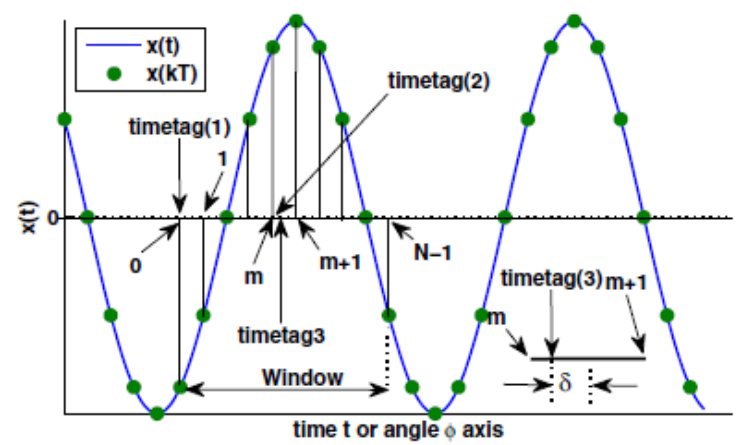

Fig. 3 Signal sampling using DFT 


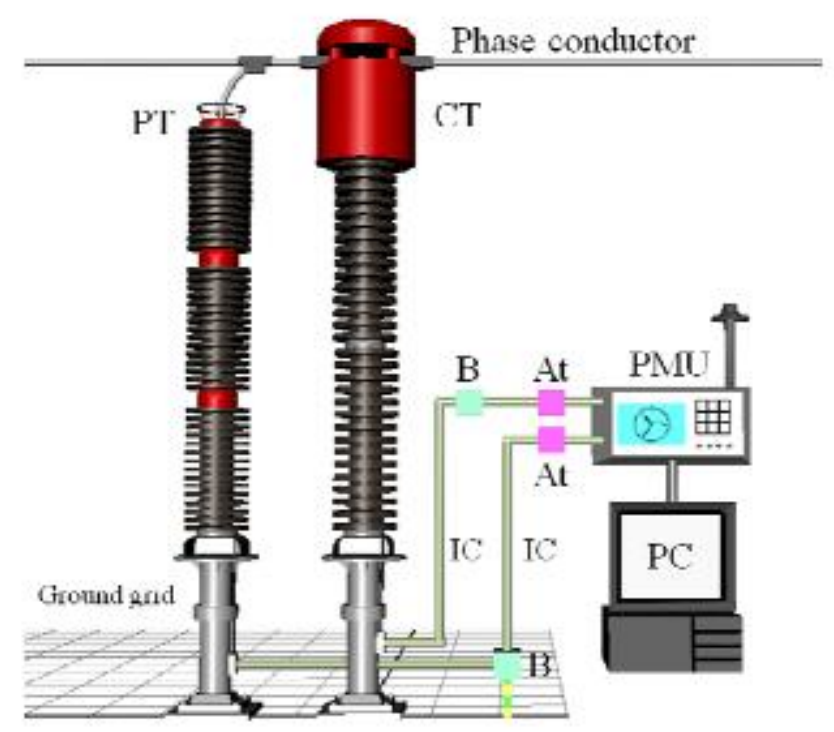

Figure 4 . PMU with GPS receiver [5]

The analog inputs are current and voltage phasors obtained from the secondary windings of the current and voltage transformers (Fig. 5).

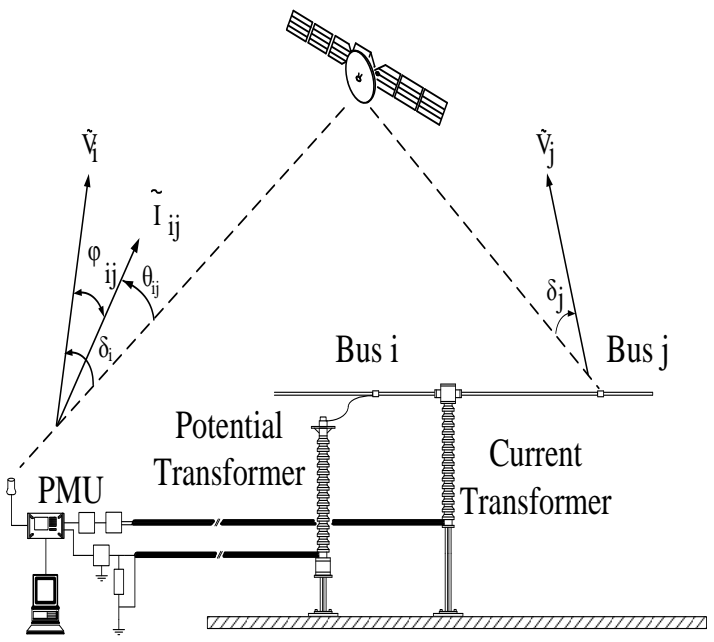

Figure 5. GPS referenced PMU at bus $i$.

\section{III.2 PHASOR MEASUREMENT UNIT}

It is assumed that each PMU has enough channels to measure the bus voltage phasor at its associated bus and the current phasors along all lines connected to that bus (Fig 6) [8].

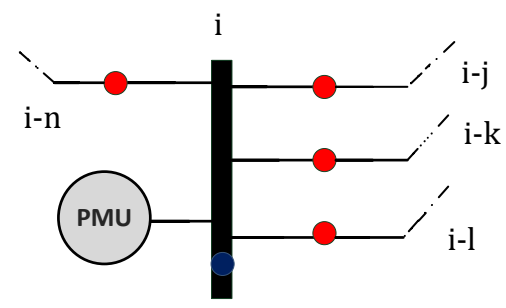

I phasor

- V phasor

Figure 6. Phasor measurements provided by a PMU installed at a network bus 
The PMUs are installed in power system substations [5]. The selection of substations where these installations take place depends upon the use of the measurements they provide. The optimal placement of PMUs will be consider in the following section. An architecture involving PMUs, communication links, and data concentration must exist in order to realize the full benefit of the Phasor measurement system. A generally accepted architecture of such system is shown in Fig.7 [5]. The devices at next level of the hierarchy are commonly known as "phasor data concentrators" (PDCs). Typical function of the PDCs is to gather data from several PMUs, reject bad data, align the time-stamps, and create a coherent record of simultaneously recorded data from a wider part of the power system. There are local storage facilities in the PDCs, as well as application functions which need the PMU data available at the PDC. This can be made available by the PDCs to the local applications in real time [5]. So, the real time data, provided by PMUs located in different regions, are transmitted over fast communication links and gathered to higher level devices, commonly known as phasor data concentrators (PDCs). Data concentrators ensure that phasor measurements are used effectively, and is a system that will retrieve files recorded at the measurement site and correlate files from different sites by the time stamp associated with each measurement. As mentioned before, a simple diagram of the phasor measurement systems hierarchy is illustrated in Fig. 7 [5]. In Fig. 7, the PMUs are suited in power system substations and provide measurements of time-stamped positive sequence voltages and currents of all monitored buses and feeders (as well as frequency and rate of frequency).

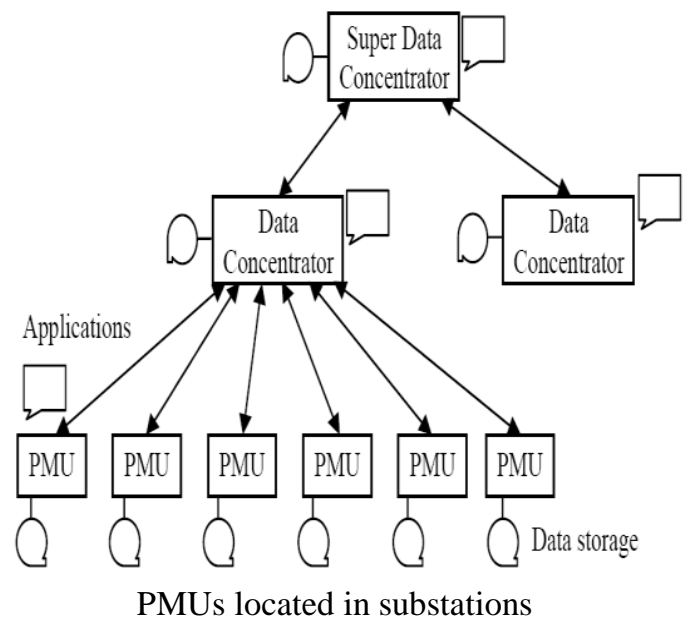

Figure 7. Hierarchy of the phasor measurement systems and levels of phasors data concentrators.

The available data are processed in control centres providing an estimate of the bus voltage phasors, the tap positions, the circuit breaker status, and other metered and unmetered electrical quantities and parameters of the power system (Fig 8).

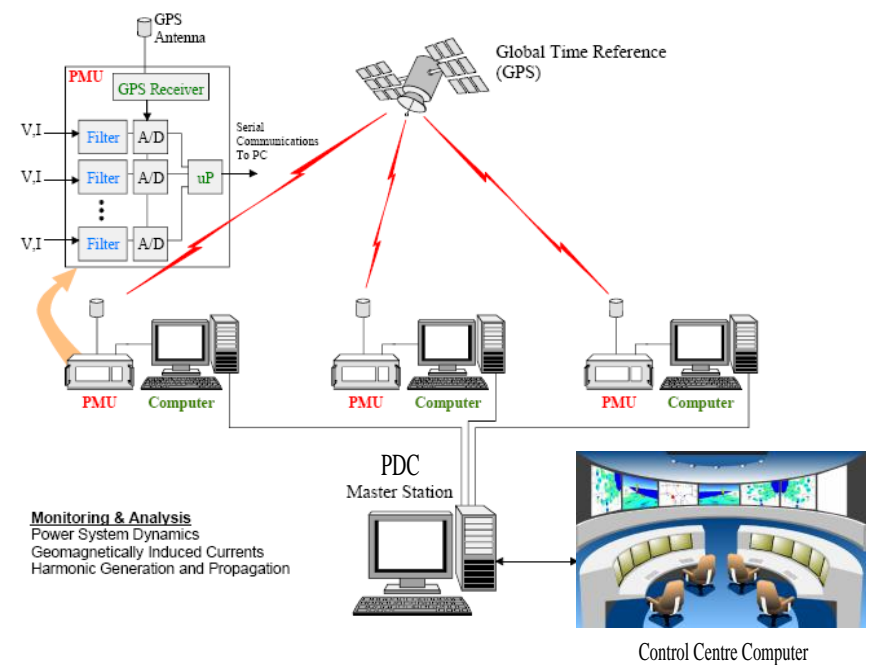

Fig 8. Phasor Data Concentrator [5] 


\section{SYNCHROPHASOR STANDARD IEEE C37.118}

Synchronized phasor measurements or synchrophasors refer to the use of a common time reference for all measured phasors. A detailed definition of a time-synchronized phasor has been codified by the IEEE C37.118 standard synchrophasor for all measured phasors. The standard defines the synchronized phasor measurements used in power systems, the methods to verify compliance with the standard and the message formats for communication with a phasor measurement unit (PMU). The phasors are tagged with a Universal Time Coordinate (UTC) time corresponding to the time of measurement [22].

\section{PMU MANUFACTURERS}

Technology for PMUs has existed for some time now and the main manufacturers are Schweitzer Engineering Laboratories (SEL), ABB, Siemens and ALSTOM GRID. Below the products are listed in the following Table I [25].

TABLE I: MODEL OF PMUS

\begin{tabular}{|c|c|}
\hline $\begin{array}{l}\text { MANUFACTURING } \\
\text { PLANT }\end{array}$ & MODEL OF PMU \\
\hline ABB & RES 521 \\
\hline \begin{tabular}{l|l|} 
Ger & ALSTOM
\end{tabular} & P847B\&C \\
\hline $\begin{array}{l}\text { AIRBITER } \\
\text { SYSTEMS } \\
\text { SYSTEM }\end{array}$ & MODEL 1133A \\
\hline EERL & TESLA 4000 \\
\hline (89.) imagination atwork & D 60 \\
\hline M macrodyne, inc. & MODEL 1690 \\
\hline SEL]SCHWETZER ENGMEERNG LABORATORES, NC, & SEL-421 \\
\hline SIEMENS & SIMEAS R-PMU \\
\hline 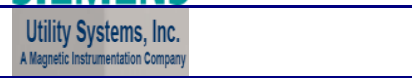 & PMU2002 \\
\hline
\end{tabular}

VI. PMU PLACEMENT OPTIMIZATION

Due to the high costs generated by the purchase and installation of a PMU, it is not feasible to locate and install the PMU at all the buses of the electric power system [7]. Therefore the objective function posed in this optimization problem focuses on minimizing the number of PMU and finding its optimal location, subject to the restriction of achieving complete observability of the network. The PMU installs at substations [5]. The dominant programming algorithm for minimizing the required PMUs is the integer linear program with binaryvalued variables. The programming model is formulated as follows :

where :

$$
\begin{aligned}
& \min J(x)=c^{T} x,\left(c_{i}=1, i=1 . . n\right) \\
& \text { s.t. } \sum_{i} a_{i j} x_{i} \geq 1 \\
& x \in\{0,1\}^{n}
\end{aligned}
$$

$$
\begin{gathered}
x_{i}=\left\{\begin{array}{l}
1, \text { if a PMU installed at bus } i \\
0 \text { otherwise }
\end{array}\right. \\
A=\left[a_{i j}\right] ; a_{i j}=\left\{\begin{array}{l}
1, \text { if } i=j \\
0 \text { otherwise }
\end{array}\right.
\end{gathered}
$$

The specific representation of the algorithm used to solve the optimal placement problem is detailed in the following Algorithm. This code solves the discrete optimization problem and uses as optimizer function the bintprog routine [26]. 


\section{TABLE II}

Algorithm : Optimal Placement of PMU

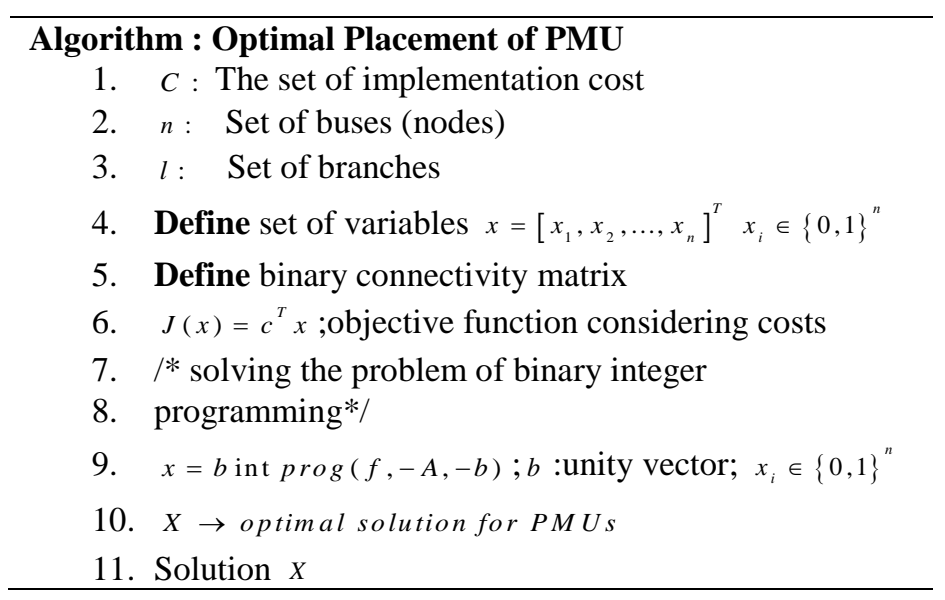

The bintprog implements a branch and bound algorithm in order to solve the discrete optimization problem [6], [26]. The Branch and Bound (BB or B\&B) algorithm is first proposed by A. H. Land and A. G. Doig in 1960 for discrete programming. It is a general algorithm for finding optimal solutions of various optimization problems, especially in discrete and combinatorial optimization. A branch and bound algorithm consists of a systematic enumeration of all candidate solutions, where large subsets of fruitless candidates are fathomed, by using upper and lower estimated bounds of the quantity being optimized [6]. The exact algorithm procedure is as below [6] :

TABLE III

IMPLEMENTATION OF BRANCH AND BOUND Algorithm

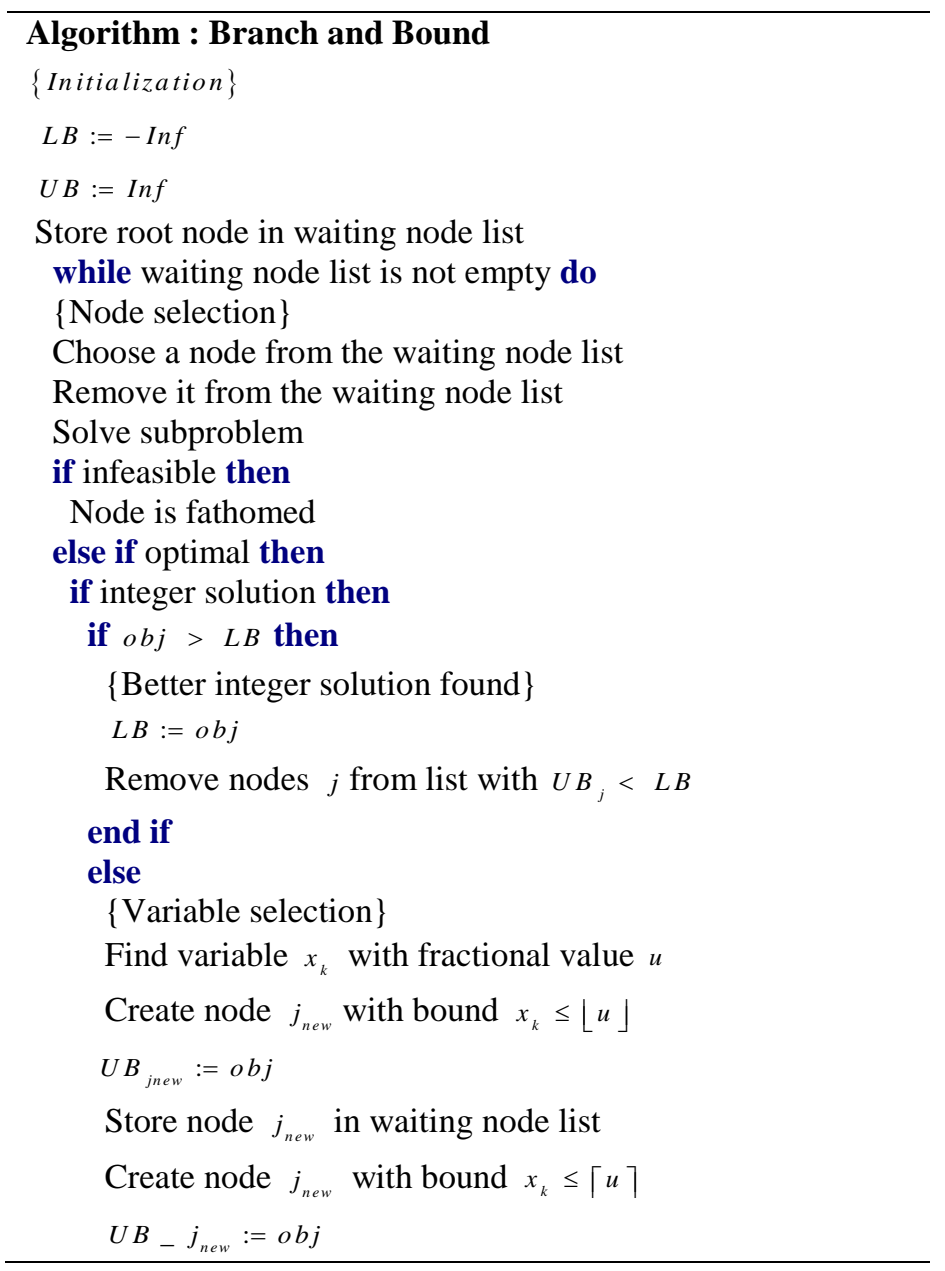


Store node $j_{\text {new }}$ in waiting node list end if

else

Stop: problem in solving subproblem

end if

$U B=\max { }_{j} U B_{j}$

end while

The flowchart of the branch and bound, the implementation of its succeed by bintprog is illustrated in Fig. 8 .

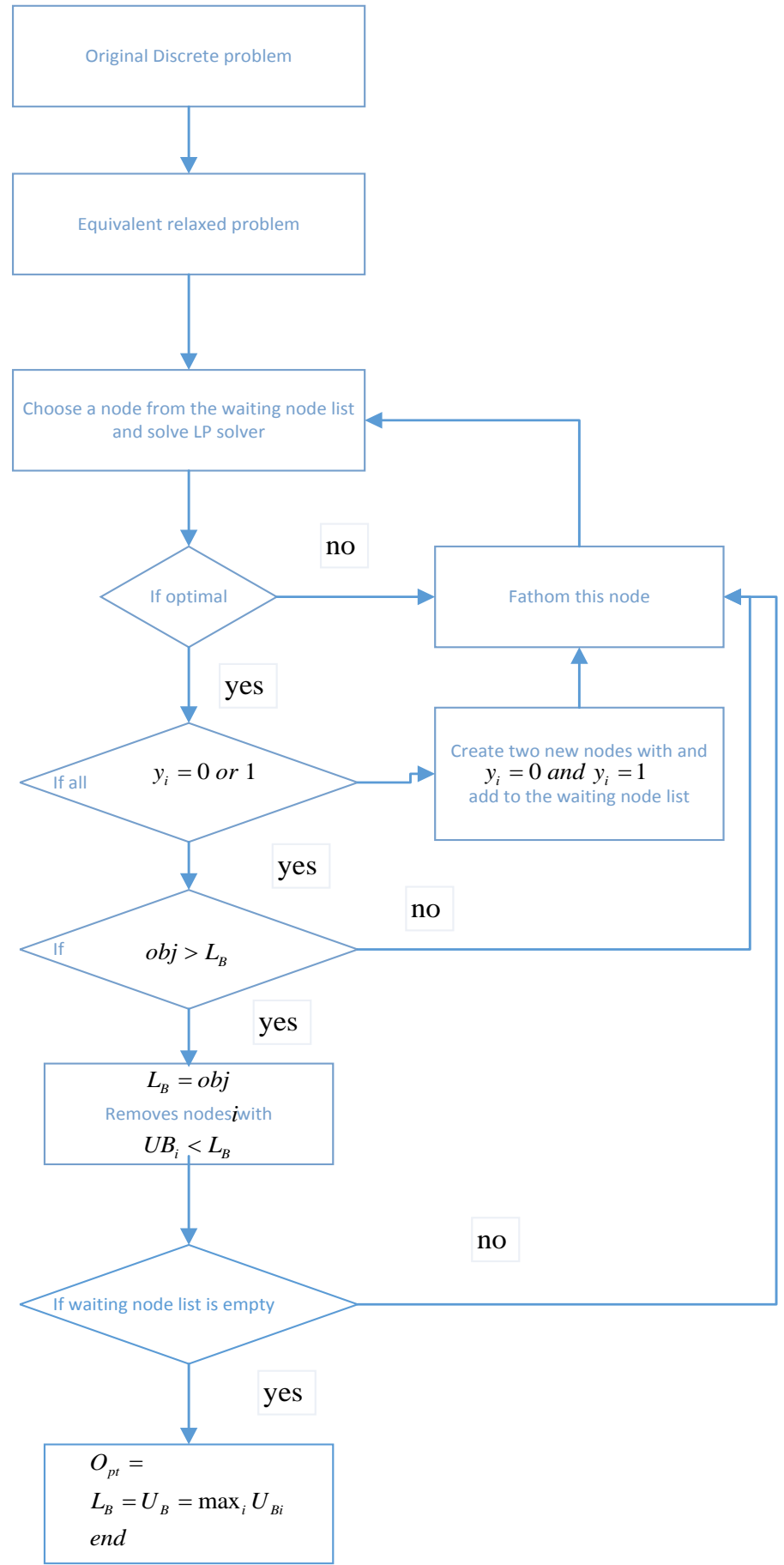

Fig. 8. Flowchart of branch and bound 


\section{CASE STUDY}

Let examine the algorithm for finding strategically PMUs at realistic power systems. Northern Regional Power Grid (NRPG) of Power Grid Corporation of India Limited (PGCIL), is the largest among all five regional power grids in India, comprising of the 9 states. It covers around 30\% geographical area and 28\% population of India. The reduced NRPG system ( $220 \mathrm{kV}$ and $400 \mathrm{kV}$ only) network only consists of 246 buses, 376 branches (lines/transformers), 42 generating units and 40 shunt reactors [28]. According with the above "Algorithm : Optimal Placement of PMU", the bintprog routine determines 70 PMUs in order to be the power system completely observable. Another realistic power system is a 129-bus test system that models the Italian HV transmission network (129-bus Italian $400 \mathrm{kV}$ transmission system (most of this information is publicly available at the GRTN web site www.grtn.it)) [29]. For the 129-bus Italian $400 \mathrm{kV}$ transmission system, the bintprog determines 40 PMUs. Another realistic power system is the Mexican power system consisting of 190 buses and 46 generators [30]. In order to be fully observable, the branch and bound through bintprog finds 59 PMUs devices. An American power system (WECC 240- bus) has been presented in [31], [32]. It is a 240-bus model as a realistic test system for the California and WECC market [31]. The numbering of the buses in this power network is not successive. The buses of each power system must be re-numbered from 1 up to the total number of buses before the simulation run of the optimization model in MATLAB. The total number of PMUs needed for full observability is 74 PMUs. The PMU locations for each realistic power system is presented in table IV.

TABLE IV: REQUIRED PMUS ENSURING COMPLETE OBSERVAB
\begin{tabular}{|l|r|}
\hline Test System & PMU location (Bus \#) \\
\hline \multirow{4}{*}{ 246 Indian } & $6,21,23,24,29,34,40,45,48,54,55,57$, \\
bus & $60,61,62,63,65,69,73,74,75,78,80,88$, \\
& $93,95,98,100,101,102,103,106,109,116$, \\
& $117,121,122,125,126,129,132134,140$, \\
& $141,142,147,157,158,160,163,168,173$, \\
& $181,183,185,187,190,191194,199,201$, \\
& $202,203,215,216,219,234,235,243,245$ \\
\hline \multirow{4}{*}{ Italian 129} & $5,28,30,33,35,37,39,43,46,47,49,50$, \\
bus & $51,52,54,55,57,59,61,64,66,69,72,73$, \\
& $74,79,80,83,92,101,103,106,107,109$, \\
\hline \multirow{4}{*}{ Mexico 190} & $110,115116,117,121,124$ \\
\hline bus & $48,49,50,53,55,56,59,60,64,77,80,81$, \\
& $83,84,86,89,90,91,96,97,99,105,106$, \\
& $107,108,109,110,111,112,117,120,122$, \\
& $128,129,131,133,136,137,138,143,145$ \\
& $146,151,153,154,158,162,164,165,168$, \\
& $170,172,174,175,176,180,185,186,189$ \\
\hline \multirow{5}{*}{ WECC $240-$} & $2,4,7,11,13,15,18,22,24,28,30,31,37$, \\
bus & $38,41,46,48,53,54,58,60,61,62,65,71$, \\
& $78,80,81,83,89,94,99,100,105,107,109$, \\
& $111,113,115,116,127,128,133146,147$, \\
& $148,149,150,156,157,160,164,165,172$, \\
& $174,177,178,180,183,184189,190,196$, \\
& $200,204,209,211,216,220,221,230,231$, \\
& 236,237 \\
\hline
\end{tabular}

\section{APPLICATION OF PMUS}

In this section, applications using synchrophasors are described. These applications can be grouped into three categories [5]:

1. Wide-area visualization and increased state awareness

2. Improved system planning and analysis

3. Response-based control applications that use real-time wide area information

4. State estimator

A very useful application for synchrophasors is Wide-Area Measurement Systems (WAMS). The WAMS refers to the possibility of observing a wide area of the electrical grid with synchrophasors. A useful application is to make a surface plot of angle measurements among PMUs located in widely dispersed location around a power system, which represents the general direction of power flows as well as the areas of sources and sinks [24]. 


\section{STATOR ESTIMATOR}

For observability analysis of systems including SCADA as well as PMU measurements, an efficient integer numerical procedure is proposed in [27] . For observability purposes, an linearized measurement vector equation is considered. $z=H x+\delta e(7)$ where $z(m \times 1)$ is the measurement vector composed of SCADA and PMU measurements, $\delta(n \times 1)$ is the vector of all bus phase angles, $H(m \times n)$ is the measurement Jacobian matrix, $e(m \times 1)$ is the measurement error vector, $m$ is the number of measurements and $n$ the number of buses. If no PMUs exist in the measurement system, then an artificial zero phase angle measurement is introduced at an arbitrarily selected reference bus, without excluding that bus from the problem formulation. It should be noted that the system observability is independent of the branch parameters as well as the operating state of the system. Therefore, all system branches can be assumed to have an impedance of $j 1.0 p . u$. and all bus voltages can be set equal to $1.0 \mathrm{p} . u$. for the purpose of observability analysis. The SCADA measurements are modeled as follows: Active power flow measurement at bus i of branch $i-j$ : active power flow at bus $i$ in branch $i-j$ can be approximated as $P_{i j}=\delta_{i}-\delta_{j}(8)$ where $\delta_{i}, \delta_{j}$ are the phase angles of the voltages at bus $i$ and $j$. An active power or zero injection at bus $i$ can be approximated as follows : $P_{i}=\sum_{j \in a(i)} P_{i j}=\sum_{j \in a(i)}\left(\delta_{i}-\delta_{j}\right)(9)$ where $a(i)$ is the set of buses connected with bus $i$. Note that $P_{i}=0$ for a zero injection at bus $i$. A PMU located at bus $i$ can measure the phase angle $\delta_{i}$ and the real part $I_{i j, \mathrm{r}}$ of the current phasor $I_{i j}$ of some or all incident branches $i-j$, which can be approximated as $I_{i j, \mathrm{r}}=\delta_{i}-\delta_{j}(10)$. The linearized Jacobian matrix is as [27] :

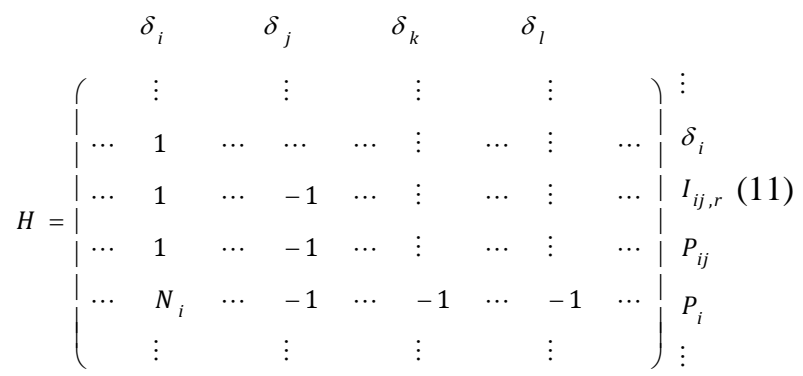

where $\mathrm{N}_{\mathrm{i}}$ is the number of incident lines at bus $i$ and $j, k, \ldots$ are the buses connected with bus $i$. The gain matrix can be formed as : $G=H^{T} \cdot H$ (12). Therefore, the power network is considered to be completely observable if and only if : the Jacobian matrix $H$ or the gain matrix $G$ has full rank, i.e, $\operatorname{rank}\left(H^{\mathrm{T}} \cdot H\right)=\operatorname{rank}(H)=n$ (13) [27], where the measurement error covariance matrix is assumed to be the identity matrix without loss of generality [27]

\section{EXISTING SYNCHROPHASOR INSTALLATIONS}

It can be said now that finally the technology of synchronized phasor measurements has come of age, and most modern power systems around the world are in the process of installing wide-area measurement systems consisting of the phasor measurement units [5]. As the initiator PMU technology, use of phasor measurement units is becoming common in electrical grids across the US. This is shown by the ambitious project NASPI and the numerous PMUs already installed in the grid. The geographical distribution of these units, in descending order, is shown in Table V.

TABLE V: Geographical area of PMUs

\begin{tabular}{|l|l|c|}
\hline A/A & \multicolumn{1}{|c|}{$\begin{array}{c}\text { Geographical area / } \\
\text { Country }\end{array}$} & $\begin{array}{c}\text { Number of } \\
\text { PMUs }\end{array}$ \\
\hline 1. & North America, China & $\mathbf{1 0 0 0}$ \\
\hline 2. & Mexico & $\mathbf{2 7 2}$ \\
\hline 3. & India & $\mathbf{1 3 0}$ \\
\hline 4. & Brazil & $\mathbf{1 0 0}$ \\
\hline 5. & $\begin{array}{l}\text { Eastern Europe, Central } \\
\text { Asia, Sibiria }\end{array}$ & $\mathbf{2 6}$ \\
\hline
\end{tabular}




\begin{tabular}{|l|l|c|}
\hline 6. & Finland & $\mathbf{2 0}$ \\
\hline 7. & Italy & $\mathbf{2 0}$ \\
\hline 8. & Spain & $\mathbf{1 3}$ \\
\hline 9. & Australia & $\mathbf{1 2}$ \\
\hline 10. & New Zeland & $\mathbf{1 0}$ \\
\hline 11. & SwEDEN, ICELAND & $\mathbf{8}$ \\
\hline 12. & Colombia & $\mathbf{6}$ \\
\hline 13. & $\begin{array}{l}\text { France, Slovenia, Croatia, } \\
\text { Switzerland }\end{array}$ & $\mathbf{5}$ \\
\hline 14. & $\begin{array}{l}\text { Denmark, Norway, } \\
\text { Poland, Hungary }\end{array}$ & $\mathbf{4}$ \\
\hline 15. & German, Slovakia & $\mathbf{3}$ \\
\hline 16. & Austria, Romania & $\mathbf{2}$ \\
\hline 17. & Belgium, Bulgaria & $\mathbf{1}$ \\
\hline $\mathbf{1 8 .}$ & Greece & $\mathbf{2}$ \\
\hline
\end{tabular}

Figure 9 shows the Union for the Co-ordination of Transmission of Electricity [25]

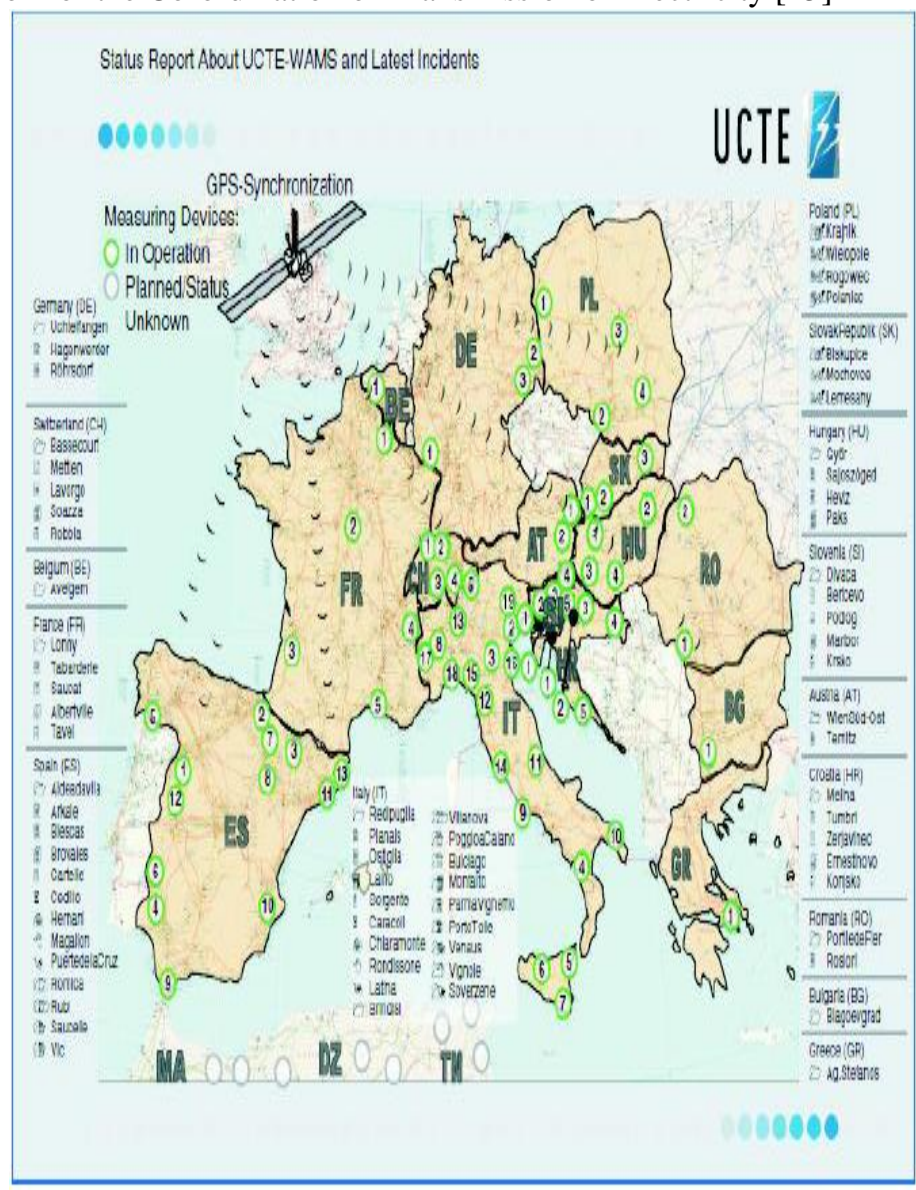

\section{PHASOR MEASUREMENT APPLICATION STUDY}

A detailed analysis has distinguished key applications and distinguished those that have a major improvement impact like angle/frequency monitoring, visualization, post-mortem analysis, model benchmarking, outage prevention, state measurement and real-time control. More advanced applications are voltage stability monitoring and state estimation using PMUs. The priorities were found to be those shown in the Figure 9. 


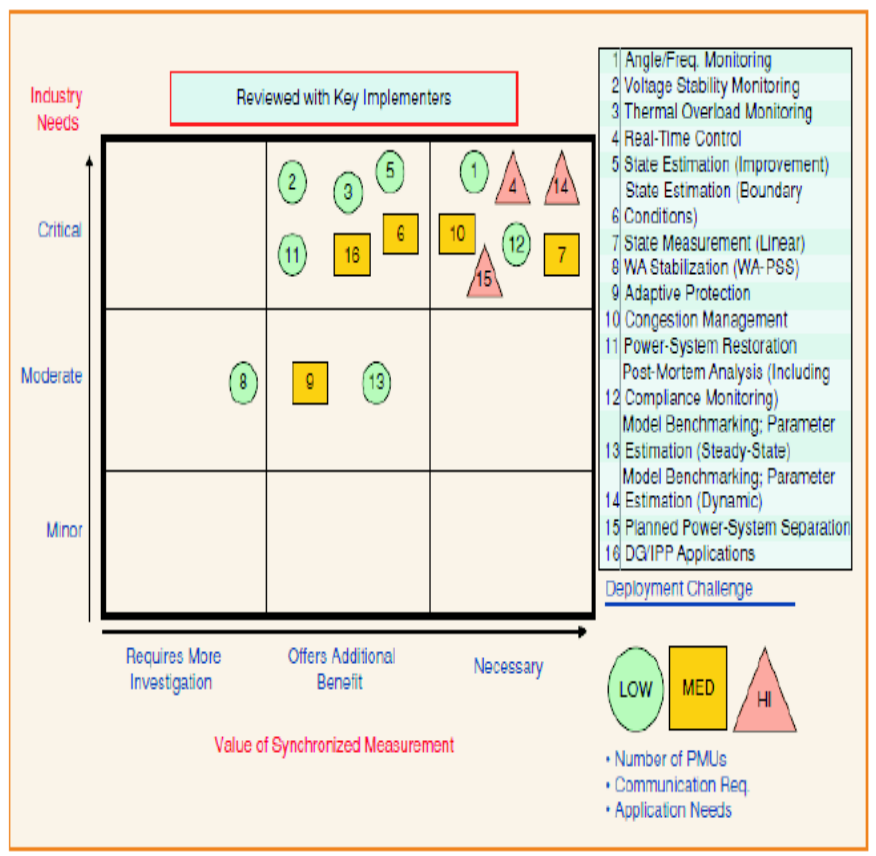

Fig. 9 PMU applications and industry needs

The CIEE study has also written a guidebook to provide general guidance for building a business case for PMU technology [25].

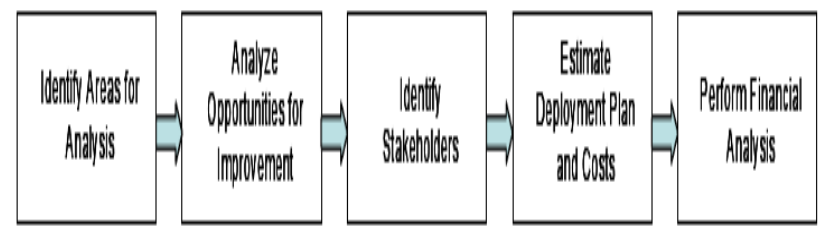

Fig 10. Business Case Analysis Process

In Figure 10, five general phases for a business case analysis for PMU technology deployment are detailed. Phase I tries to understand performance gaps with existing technologies and practices and understanding what the added value of the PMU would be. Phase II analyses the issues that can be addressed by PMU technology. Phase III identifies the stakeholders and the benefits for them of the PMUs. Phase IV establishes an expected plan for PMU deployment. Finally, phase V compares projected benefits over a time horizon. The applications are separated in new-term (1-3 years), medium-term (3-5 years) and long-term (more than 5 years). The traditional approach to evaluate the project is to calculate the NPV (Net Present Value), where all future benefits, expenses and needed investment for a number of years is included in the calculation (TABLE VI)

TABLE VI: NPV estimations for different uses of PMUs

\begin{tabular}{|c|c|}
\hline Case & NPV (1.000€) \\
\hline 1. Full deployment & $43.666 \varepsilon \dot{\varepsilon} \omega \varsigma 317.759$ \\
\hline 2. Post-mortem analysis only & $\dot{\varepsilon} \omega \varsigma 78$ \\
\hline $\begin{array}{l}\text { 3. Outage Prevention and Post- } \\
\text { mortem analysis only }\end{array}$ & $19.023 \varepsilon \dot{\varepsilon} \omega \varsigma 314.932$ \\
\hline 4. Congestion management only & 1.258 \\
\hline
\end{tabular}

As shown by Table VI, full deployment of all applications is most beneficial since the same capital investment can be used by different applications. The study concluded that there are large potential benefits of synchronized phasor measurement technology, such as improvement of safety, reliability and efficiency of the grid as well as large reliability and financial benefits for all grid users. The challenge is to move from the research and 
development of PMUs and their applications to commercial operation. As the study has shown, phasor measurement capability is advanced enough for commercial implementation to be possible. The difficulty is that business processes and models for the implementation of PMUs have not been developed in most companies so that the move to PMU technology and its applications is restricted. Due to the significant costs in infrastructure and technology for PMU projects, identification of quantifiable benefits can facilitate acceptance and funding of the projects. These benefits fall in the tangible and intangible categories. Tangible benefits are the better utilization of system capacity, more efficient use of manpower and improved reliability of operations. However, most benefits are not so tangible. These include avoided costs associated with outage investigation, blackout restoration costs, costs of regulation following major system events [25].

\section{FINALLY REMARKS}

Synchrophasor technology is an innovative measurement technique which is very useful for power system operation and control. In many countries, synchrophasor technology is still in the research and development phase. As has been shown in this paper, an international standard has been presented for synchrophasors measurements and many applications have already been presented for these PMUs. Synchrophasor technology has already been adopted by many countries around the world, and this has been described extensively in this paper. Various companies have worked on Phasor Measurement Units and also have manufactured Phasor Data Concentrators, which allow the processing of many phasor measurements for power system analysis and control. Due to the high cost of a PMU device, it is crucial an optimal allocation of PMUs in power networks. A branch and bound algorithm has been presented in order to solve this combinatorial optimization problem. Therefore, PMU technology is being implemented in grids around the world for mainly analyzing wide-area phenomena and better and reliable monitoring of power system.

\section{ACKNOWLEDGMENT}

The author is grateful to his professor Nicholas G. Maratos at the School of Electrical and Computer engineering of NTUA for his support to the author's studies at the university and his valuable advices about optimization techniques. This work was supported by Greeks national funds under a scholarship provided by ELKE -NTUA (http://edeil.ntua.gr/) to the author for postgraduate studies and research at the university.

\section{REFERENCES}

[1] A. Monticelli, Generalized State Estimation, Kluwer Academic Publishers, 1999

[2] IEEE Standard for Synchrophasors for Power Systems, IEEE Standard C37.118-2005, Jun. 2005.

[3] J. S. Thorp, A. G. Phadke, and K. J. Karimi, "Real time voltage-phasor measurements for static state estimation”, IEEE Trans. Power App. Sys., vol. 104, no. 11, pp. 3098-3106, Nov. 1985.

[4] A. G. Phadke, J. S. Thorp, and K. J. Karimi, "State estimation with phasor measurements," IEEE Trans. Power Syst., vol. 1, no. 1, pp. 233-241, Feb. 1986.

[5] A. G. Phadke and J. S. Thorp, "Synchronized Phasor Measurements and Their Applications", New York: Springer, 2008

[6] X.S Yang "Engineering Optimization : An introduction with Metaheuristic Applications, “ 2010, John Willey and Sons, Inc

[7] N. M. Manousakis, G. N. Korres, and P. S. Georgilakis, "Taxonomy of PMU Placement Methodologies.", IEEE Trans. Power Syst., vol. 27, no. 2, pp. 1070-1077, May 2012.

[8] B. Xu, and A. Abur, "Observability analysis and measurement placement for systems with PMUs," in Proc. IEEE Power Eng. Soc. Power Systems Conf. Exposition, Oct. 2004, pp. 943-946.

[9] B. Gou, "Optimal placement of PMUs by integer linear programming," IEEE Trans. Power Syst., vol. 23, no. 3, pp. 1525-1526, Sep. 2008.

[10] N.P Theodorakatos., N.M Manousakis and Korres, "Optimal placement of PMUs in power systems using binary integer programming and genetic algorithm“, 9th Mediterranean Conference on Power Generation, Transmission Distribution and Energy Conversion, MedPower 2014; Athens; Greece, (12), pp. $140-146$ 
[11] D. Dua, S. Dambhare, R. K. Gajbhiye, and S. A. Soman, "Optimal multistage scheduling of PMU placement: An ILP approach,” IEEE Trans. Power Del., vol. 23, no. 4, pp. 1812-1820, Oct. 2008.

[12] N.P Theodorakatos., N.M Manousakis and Korres "Optimal PMU Placement Using Nonlinear Programming, " OPT-i-An International Conference on Engineering and Applied Sciences Optimization, 2014, Kos Island, Greece, pp. 240-258

[13] S. Chakrabarti, E. Kyriakides, and D. G. Eliades, "Placement of synchronized measurements for power system observability,” IEEE Trans. Power Del., vol. 24, no. 1, pp. 12-19, Jan. 2009

[14] N.P Theodorakatos., N.M Manousakis and Korres " A sequential quadratic programming method for contingency constrained phasor measurement unit placement", International Transactions on Electrical Energy Systems , 2015, 25, (12), pp. 3185- 3211

[15] N.P Theodorakatos., N.M Manousakis and Korres " Theodorakatos N.P, Manousakis N.M, and Korres G.N.: Optimal Placement of Phasor Measurement Units with Linear and Non- linear Models, “ Electric Power Components and Systems, 2015, 43, (4), pp. 357-373

[16] A. Ahmadi, Y. Alinejad-Beromi, and M. Moradi, "Optimal PMU placement for power system observability using binary particle swarm optimization and considering measurement redundancy," Expert Systems with Applications, vol. 38, pp. 7263-7269, 2011

[17] S Chakrabarti., and E Kyriakides, "Optimal placement of phasor measurement units for power system observability, " IEEE Trans. Power Syst., 2008, 23, (3), pp. 1433-1440

[18] S Chakrabarti., G Venayagamoorthy, and E Kyriakides, "PMU placement for power system observability using particle swarm optimization“, 2008, Australasian Univ. Power Eng. Conf

[19] J Sun Peng, , Y and Wang H.F, “ Optimal PMU placement for full network observability using Tabu search algorithm“, Elec. Power Syst. Res., 2006, 28, (4), pp. 223-231

[20] N. C Koutsoukis, N. M Manousakis., P. S Georgilakis,., and G. N Korres, "Numerical observability method for optimal phasor measurement units placement using recursive Tabu search method", IET Generation, Transmission and Distribution., 2013, 7, (4), pp. 347-356

[21] CERTS. Phasor technology and real-time dynamics monitoring system (rtdms). Technical report, Electric Power Group, 2006

[22] Power System Relaying Committee. IEEE standard for synchrophasors for power systems. Technical report, IEEE Power Engineering Society, 2005

[23] M. Karimi-Ghartemani, O. Boon-Teck, and A. Bakhshai, "Investigation of DFT-based phasor measurement algorithm“. In Power and Energy Society General Meeting, 2010

[24] R.F. Nuqui. State estimation and Voltage Security Monitoring Using Synchronized Phasor Measurements. PhD thesis, Virginia Polytechnic Institute and State University, 2001

[25] M. Hurtgen and J-C. Maun, "Applications of PMU measurements in the Belgian electrical grid“, 2012

[26] MATLAB. The MathWorks Inc., Natick, Massachusetts

[27] G.N Korres, "An integer-arithmetic algorithm for observability analysis of systems with SCADA and PMU measurements', Electric Power Systems Research 81 (2011) 1388-1402.

[28] Online. [Available] :http://www.iitk.ac.in/eeold/facilities/Research_labs/Power_System/NRPGDATA.pdf

[29] F. Milano_, C.A. Canizares, M. Invernizzi, "Multiobjective optimization for pricing system security in electricity markets",IEEE Transactions on Power Systems, 18, (2), 2003

[30] J.M Ramirez,., B.V Hernandez., R.E Correa, “ Dynamic equivalence by an optimal strategy“, Electric Power Systems Research 84 (2012), 2011, p.p 58- 64

[31] J.E. Price and J. Goodin, " Reduced network modeling of WECC as a market design prototype, " In Power and Energy Society General Meeting, 2011 IEEE, pages 1 -6, Jul. 2011.

[32] James Price, 240 Bus WECC Model Data. [Online]. Available: www.pserc.wisc.edu/documents/publications/reports/2010_reports/WECC240\%20data\%20for\%20IEE E\%20PES\%202011GM0942.zip 


\section{AUTHOR'S BIOGRAPHY}

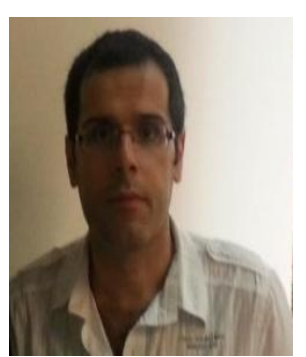

Nikolaos P Theodorakatos received his B.S. and his Diploma degrees in electrical engineering from Technological Educational Institute of Athens and National Technical University of Athens, Athens, Greece, in 2000 and 2012, respectively. Currently, he is pursuing his Ph.D. at the School of Electrical Engineering of National Technical University of Athens, Athens, Greece. His research interests include PMU technology and optimization techniques, mathematical programming and Control of Power Systems 